\title{
Severity of core symptoms in first episode schizophrenia and long-term remission
}

\author{
Carlo Marchesi ${ }^{\mathrm{a}, \mathrm{b}, *}$, Andrea Affaticati $^{\mathrm{b}}$, Alberto Monici ${ }^{\mathrm{b}}$, Chiara De Panfilis ${ }^{\mathrm{a}, \mathrm{b}}$, \\ Paolo Ossola ${ }^{\mathrm{a}}$, Matteo Tonna ${ }^{\mathrm{b}}$ \\ a Department of Neuroscience, Psychiatric Unit, University of Parma, Parma, Italy \\ ${ }^{\mathrm{b}}$ Mental Health Department, Local Health Service, Parma, Italy
}

\section{A R T I C L E I N F O}

\section{Article history:}

Received 6 November 2013

Received in revised form

7 October 2014

Accepted 3 November 2014

Available online 11 November 2014

Keywords:

First-episode schizophrenia

Long-term remission

Core symptoms

\begin{abstract}
A B S T R A C T
A definable concept of symptomatic remission in schizophrenia has been proposed by the Remission in Schizophrenia Working Group (RSWG). Nevertheless no studies to date assessed eventual differences in core symptoms at onset between remitters and non-remitters. The present study evaluated whether the severity of core symptoms differed among 48 patients with first episode schizophrenia (FES), and whether it predicted long-term (16-years) remission. Particularly, the present study aimed to verify if RSWG remission criteria might identify a sub-group of patients with mild core symptoms at their first episode. In the present study the severity of core symptoms was significantly lower in remitted than in non-remitted patients; interestingly, five out of the eight core symptoms already satisfied the severity criteria for remission in most remitted patients. Among the core symptoms only the severity of social withdrawal predicted the long-term outcome, while age at onset, duration of untreated psychosis and employment status did not exert any effect. Concluding, patients with FES presenting, mild core symptoms, particularly low negative symptoms, were more likely to reach long-term remission. Therefore, RSWG remission criteria seem to identify a subgroup of FES patients with mild severe core symptoms so with a higher probability to reach remission.
\end{abstract}

(c) 2014 Elsevier Ireland Ltd. All rights reserved.

\section{Introduction}

Since the description of dementia praecox (Kraepelin, 1899, schizophrenia has been conceptualized as a chronic illness and remission has long been considered largely impossible, with the consequence that diagnosis and prognosis were viewed as essentially the same (McGlashan, 2008). However, in the recent years the Remission in Schizophrenia Working Group (RSWG) (Andreasen et al., 2005) posited that "symptomatic remission is a definable concept and an achievable stage in the treatment of schizophrenia" and proposed criteria for symptomatic remission. According to such criteria, remission is defined as: "a state in which patients have experienced an improvement in core symptoms, to the extent that these symptoms no longer interfere significantly with daily life". Core symptoms are those perceived as diagnostically characteristics for the condition, and eight items of the Positive and Negative Syndrome Scale (PANSS) were

\footnotetext{
* Corresponding author at: Università di Parma, Dipartimento di Neuroscienze, Unità di Psichiatria, Ospedale-Padiglione Braga, Via Gramsci 14, Parma 43125, Italy. Tel.: + 390521 903594; fax: + 390521347047.

E-mail address: carlo.marchesi@unipr.it (C. Marchesi).
}

proposed for their evaluation. Remission is achieved if all eight items are rated as mild (Andreasen et al., 2005).

According to these criteria a substantial proportion of patients with schizophrenia (45-70\%) (Lambert et al., 2010) can be defined as remitters at some point during the course of their illness. However, patients who eventually reach the RSWG remission condition may be highly heterogeneous. For instance, remitted patients could have shown, at illness onset, a different severity of the core symptoms when compared to patients who will not achieve remission, even though no studies have specifically investigated this topic.

Therefore, the present study aimed to evaluate whether 1) patients with first episode schizophrenia (FES) who gained remission after several years of illness differed from non-remitters with respect to the severity of the core symptoms, and 2) which of the core symptoms predicted long-term remission. Particularly, we aimed to verify if RSWG remission criteria identified a sub-group of patients with mild core symptoms at their first episode.

\section{Methods}

The study protocol was approved by the local ethic committee. This study is a part of a more extensive evaluation of outcome in patients with a first psychotic 
episode and data on long-term outcome have been already published (Marchesi et al., 2014).

\subsection{Sample}

The study participants were recruited from patients who were consecutively admitted to the Psychiatric Clinic of the University of Parma, from January 1995 to December 1999, for the first psychotic episode. A physician or a psychiatrist requested the admission for the onset of delusions, hallucinations or behavior disorganization.

Patients were included in the study if: 1 ) they were aged over 17 years; 2 ) they were hospitalized for the first time in a psychiatric unit for a first psychotic episode; 3 ) they were discharged with a diagnosis of Schizophrenia, according to the DSM-IV; 4) they accepted to be revaluated after many years from the index admission; and 5) they gave a written informed consent during the index hospitalization.

Patients were excluded from the study if: 1) they were affected by drug abuse or drug dependence, delirium, mental retardation or organic mental disorders; 2) they were previously treated with psychotropic medication; 3) they did not assure treatment adherence or they discontinued the treatment program after discharge from hospital for more than two consecutive visits (see follow-up evaluation).

\subsection{Assessment}

\subsubsection{Baseline assessment}

The baseline evaluation was carried out within the first week of hospitalization. Socio-demographic variables recorded at baseline were: age, gender, years of education, marital and employment status, housing situation, age at onset of schizophrenia and the time passed from the onset of psychotic symptoms or behavioral changes to the time of hospitalization (i.e., when patients received treatment for the first time).

The diagnosis of schizophrenia was assessed by means of the Structured Clinical Interview for Axis I DSM-IV disorders, carried out by trained psychiatrists.

Symptom severity was measured with the Positive and Negative Syndrome Scale (PANSS).

\subsubsection{Follow-up evaluation}

After hospital discharge, patients accessed to continuous and, reasonably comprehensive, public mental health services (Amaddeo et al., 2012), where they attended nearly monthly visits.

During each visit their psychopathological condition, treatment adherence, medications prescription, and use of illicit drugs were clinically monitored and these data were freely accessible from the therapists over the 16 year follow-up.

Patients were re-examined in 2010 by a psychiatrist, blind to the baseline evaluation. The follow-up assessment consisted in the administration of SCID-I and PANSS. After eight months the patients were re-evaluated with the PANSS in order to determine whether the time criterion of 6 months requested by RSWG for remission was satisfied. Only after the follow-up assessment, patients were defined in remission ( $\mathrm{R}$ ) or in non-remission (NR), according to both severity and time criteria proposed by the RSWG.

\subsection{Treatment}

All patients received antipsychotic medications. In the effort to maximize treatment adherence, a medication control by relatives was recommended and the count of pills was performed at any time the medication was prescribed. The use of long-acting compounds was proposed to patients who did not assure treatment adherence.

\subsection{Data analysis}

Before the start-up of the study and the follow-up evaluations an inter-rater reliability was calculated for the diagnosis of schizophrenia (baseline: $k=0.92$; follow-up: $k=0.95$ ) and for the PANSS core symptoms scores (baseline: $k=0.72-$ 0.86; follow-up: $k=0.73-0.90$ ).

The clinical and socio-demographic features were compared in R and in NR using two-tailed Student's $t$-test for continuous variables and Fischer's exact test for categorical variables.

Lastly, a logistic regression analysis (a stepwise method) was applied to evaluate whether core symptoms severity at baseline together with gender, age at onset, DUP and working status (independent variables) predicted the remission status at follow-up (dependent variable: R vs NR).

The statistical software IBM SPSS Statistics 20 version was used for the analyses. Statistical significance was set at the level of $p \leq 0.05$.

\section{Results}

\subsection{Sample}

During the period of recruitment, 75 patients with FES were consecutively admitted to the Psychiatric Clinic. Twenty-seven patients were not included in the study: eight were lost at followup; three refused to be revaluated; 16 were non-adherent to the monthly visits or treatment and therefore they were excluded from the study. The remaining 48 patients participated in the study and were re-evaluated in 2010.

The SCID-IV re-administration confirmed the diagnosis of schizophrenia in all 48 patients. None of them was using illicit drugs.

Eighteen patients (37.5\%) satisfied the remission RSWG criteria $(\mathrm{R})$, while the remaining 30 patients (62.5\%) did not (NR). Among remitted patients, three of them $(16.7 \%)$ were in complete symptomatic recovery, nine (50\%) showed minimal symptom severity and six (33.3\%) had mild symptom severity at follow-up.

The socio-demographics and clinical features at baseline were reported in Table 1.

At the follow-up $\mathrm{R}$ and NR patients showed similar age (R: $40.5 \pm 9.8$ yrs; NR: $44.8 \pm 11.7$ yrs $)(t=1.3 ; p=0.19)$ and duration of illness (R: $16.0 \pm 4.8$ yrs; NR: $16.3 \pm 5.1$ yrs) ( $t=0.1$; $p=0.85$ ), while the number of psychotic episodes requiring hospitalization were higher in NR $(3.6 \pm 1.0)$ than in $\mathrm{R}(2.2 \pm 0.6)$ $(t=4.7 ; p<0.001)$.

\subsection{Core symptoms severity}

At baseline, the severity of core symptoms was lower in $\mathrm{R}$ than in NR (Table 1). Furthermore, the severity criterion for remission (item score $\leq 3$ ) was already satisfied for unusual thought content, blunted affect and social withdrawal more frequently in $\mathrm{R}$ than in NR patients. Lastly, five out of the eight core symptoms already satisfied the severity criterion for remission in most $\mathrm{R}$ patients $(n=11 ; 61 \%)$ but only in a minority of NR patients $(n=3 ; 10 \%)$.

\subsection{Core symptoms severity and prediction of remission}

At baseline, among the core symptoms only the severity of social withdrawal predicted the long-term outcome: this core symptom was negatively associated with remission status at follow-up $(\beta=-2.1$; wald $=11.7 ; \quad O R=0.11 ;$ CI $95 \%=0.03-0.040$; $p=0.001$ ). Moreover, gender, age at onset, DUP and working status did not exert any effect on long-term symptomatic remission.

\section{Discussion}

In the present study patients with schizophrenia were evaluated with the PANSS at their first episode of illness and 16 years thereafter.

At the time of their first episode of illness, patients who reached remission during the follow-up ( $R$ patients), showed a lower severity of almost all core symptoms than NR; in fact, only the severity of hallucinations and conceptual disorganization was comparable in R and in NR. Interestingly, if applying the severity criteria of the RSWG at the first episode, most of the core symptoms (unusual thought content, mannerisms, conceptual disorganization, blunted affect and social withdrawal) would have already satisfied such criteria in the majority (50-88\%) of $R$ patients, whereas only mannerism would have satisfied this criterion in most (53\%) NR. In other words, in R patients the first episode was mainly characterized by delusions, hallucinations and 
Table 1

Socio-demographic and symptoms features at baseline in patients with first episode schizophrenia.

\begin{tabular}{|c|c|c|c|c|c|c|}
\hline & \multicolumn{4}{|c|}{ Remission at follow-up } & \multirow[b]{3}{*}{$F^{a}$} & \multirow[b]{3}{*}{$\boldsymbol{p}$} \\
\hline & \multicolumn{2}{|c|}{$\begin{array}{l}\text { Yes } \\
\text { n. } 18\end{array}$} & \multicolumn{2}{|c|}{$\begin{array}{l}\text { No } \\
\text { n. } 30\end{array}$} & & \\
\hline & $\boldsymbol{n}$. & $\%$ & $\boldsymbol{n}$. & $\%$ & & \\
\hline \multicolumn{7}{|l|}{ Gender } \\
\hline Male & 10 & 55.5 & 19 & 63.3 & 0.2 & 0.76 \\
\hline Marital status & & & & & 3.6 & 0.19 \\
\hline Never married & 13 & 72.2 & 22 & 73.3 & & \\
\hline Married & 5 & 27.8 & 4 & 13.3 & & \\
\hline Divorced/Widowed & 0 & 0.0 & 4 & 13.3 & & \\
\hline Working status & & & & & 6.4 & 0.01 \\
\hline Never occupied & 4 & 22.2 & 18 & 60.0 & & \\
\hline Occupied & 14 & 77.8 & 12 & 40.0 & & \\
\hline Living status & & & & & 0.5 & 0.54 \\
\hline Living alone & 8 & 44.4 & 10 & 33.3 & & \\
\hline \multirow[t]{2}{*}{ Living with someone } & 10 & 55.6 & 20 & 66.7 & & \\
\hline & \multicolumn{2}{|c|}{ mean \pm S.D. } & \multicolumn{2}{|c|}{ mean \pm S.D. } & $t$ & $\boldsymbol{p}$ \\
\hline Age at onset years & \multicolumn{2}{|c|}{$22.2 \pm 3.8$} & \multicolumn{2}{|c|}{$21.6 \pm 5.8$} & 0.3 & 0.70 \\
\hline DUP weeks & \multicolumn{2}{|c|}{$18.0 \pm 4.5$} & \multicolumn{2}{|c|}{$19.7 \pm 4.4$} & 1.2 & 0.20 \\
\hline \multicolumn{7}{|l|}{ PANSS scores } \\
\hline Positive score & \multicolumn{2}{|c|}{$24.8 \pm 3.7$} & \multicolumn{2}{|c|}{$28.1 \pm 4.0$} & 2.7 & 0.008 \\
\hline Negative score & \multicolumn{2}{|c|}{$17.8 \pm 3.1$} & \multicolumn{2}{|c|}{$25.3 \pm 4.4$} & 6.1 & $<\mathbf{0 . 0 0 1}$ \\
\hline General psychopathology score & \multicolumn{2}{|c|}{$46.0 \pm 4.1$} & \multicolumn{2}{|c|}{$55.8 \pm 5.4$} & 6.6 & $<\mathbf{0 . 0 0 1}$ \\
\hline Total score & \multicolumn{2}{|c|}{$88.8 \pm 6.2$} & \multicolumn{2}{|c|}{$109.2 \pm 10.0$} & 7.7 & $<\mathbf{0 . 0 0 1}$ \\
\hline \multicolumn{7}{|l|}{ PANSS core items score } \\
\hline Delusions (P1) & \multicolumn{2}{|c|}{$4.7 \pm 0.7$} & \multicolumn{2}{|c|}{$5.3 \pm 0.8$} & 2.4 & 0.02 \\
\hline Hallucinations (P3) & $3.9=$ & 1.3 & 4.2 & 1.4 & 0.6 & 0.50 \\
\hline Unusual thought Content (G9) & $3.5=$ & 0.7 & 4.4 & 0.9 & 3.2 & 0.002 \\
\hline Conceptual disorganization (P2) & 3.5 & 1.3 & 3.8 & 0.9 & 0.9 & 0.32 \\
\hline Mannerisms/posturing (G5) & 2.7 & 0.8 & 3.3 & 0.9 & 2.3 & 0.02 \\
\hline Blunted affect (N1) & $3.0=$ & 0.6 & 3.6 & 0.8 & 2.8 & 0.007 \\
\hline Social withdrawal (N4) & 2.7 & 0.6 & 4.1 & 0.8 & 5.5 & $<\mathbf{0 . 0 0 1}$ \\
\hline Lack of spontaneity (N6) & 1.8 & & 3.1 & 0.9 & 4.7 & $<0.001$ \\
\hline PANSS score $\leq \mathbf{3}$ (yes) & $n$. & $\%$ & $\boldsymbol{n}$. & $\%$ & $F^{a}$ & $\boldsymbol{p}$ \\
\hline Delusions (P1) & 1 & 5.6 & 1 & 3.3 & 0.1 & 1.00 \\
\hline Hallucinations (P3) & 6 & 33.3 & 8 & 26.7 & 0.2 & 0.74 \\
\hline Unusual thought content (G9) & 9 & 50.0 & 5 & 16.7 & 6.0 & 0.02 \\
\hline Conceptual disorganization (P2) & 9 & 50.0 & 8 & 26.7 & 2.6 & 0.12 \\
\hline Mannerisms/posturing (G5) & 15 & 83.3 & 16 & 53.3 & 4.4 & 0.06 \\
\hline Blunted affect (N1) & 14 & 77.8 & 11 & 36.7 & 7.6 & 0.008 \\
\hline Social withdrawal (N4) & 16 & 88.9 & 7 & 23.3 & 19.3 & $<0.001$ \\
\hline Lack of spontaneity (N6) & 15 & 83.3 & 16 & 53.3 & 4.4 & 0.06 \\
\hline$\geq 5$ Items with a score $\leq 3$ & 11 & 61.1 & 3 & 10.0 & 17.4 & $<0.001$ \\
\hline
\end{tabular}

${ }^{a}$ Fischer's exact test.

behavioral disorganization; thus they might present a mild severity of the other core symptoms.

Indeed, the severity of the negative symptoms specifically differentiated $\mathrm{R}$ and NR at the first episode, since blunted affect, social withdrawal and lack of spontaneity showed a lower severity in R than in NR. Further, the core symptoms "blunted affect" and "social withdrawal" satisfied the severity criteria for remission in $77-88 \%$ of $\mathrm{R}$ patients and in 36-23\% in NR at illness onset. Finally, among the core symptoms, only the severity of social withdrawal at first episode negatively predicted 16-year, long-term remission. In a previous long-term study (Marchesi et al., 2014) negative symptoms, already present in FES patients, remained stable thorough the course of the illness, could not be considered as a consequence of a chronic course, and exerted a negative effect on the achievement of remission. This finding is in keeping with recent studies indicating an association between higher negative symptoms at first episode and poor outcome (Kurihara et al., 2011; Ayesa-Arriola et al., 2013; Lang et al., 2013).
This finding is in line with classical psychopathology, which consider negative dimension as "fundamental" for the outcome of schizophrenia (Kraepelin, 1899; Bleuler, 1911) probably implying specific etio-pathogenetic pathways (Crow, 1985; Kirkpatrick et al., 2001).

Interestingly, $\mathrm{R}$ and $\mathrm{NR}$ patients differed significantly about working status at the baseline, with a lower rate of NR patients being employed. This finding confirms that a worse premorbid social functioning is associated with a non-remission (Lambert et al., 2006; San et al., 2007; Wunderink et al., 2009; Karow et al., 2012) and supports the suggestion that premorbid impairment in social functioning may represent a precursor of negative symptoms (Strous et al., 2004; Allen et al., 2005; Chang et al., 2013). Therefore, we can hypothesize that in our patients poor premorbid social functioning can facilitate the onset of negative symptoms, which in turn may reduce the likelihood to reach a symptomatic remission.

Concerning positive core symptoms, $\mathrm{R}$ patients exhibited more positive than negative symptoms at their first episode. However, logistic regression analysis demonstrated that the likelihood to develop remission is not influenced by the severity of positive symptoms, suggesting that positive symptoms at first episode do not have impact on later symptomatic remission. In a previous long-term study (Marchesi et al., 2014) positive symptoms showed a fluctuating course and their prognostic value depended more on their recurrence than of their initial severity.

Taken together, these findings suggest that: (1) patients who achieved remission at follow-up presented a mild severity of the core symptoms at their first episode of illness; (2) specifically, most $\mathrm{R}$ patients already showed, at illness onset, the severity of the core symptoms requested for remission and (3) among the core symptoms, social withdrawal seems to negatively influence the achievement of remission.

Thus, the results of the present study suggest that at first episode lesser symptoms severity, particularly a lesser degree of negative symptoms, predict long-term remission in schizophrenic patients, confirming the data of previous studies with a shorter period of follow-up (Lasser et al., 2007; Novick et al., 2007; Addington and Addington, 2008; Rossi et al., 2009; Ventura et al., 2009; Lambert et al., 2010; Buoli et al., 2012; Ayesa-Arriola et al., 2013; Lang et al., 2013). In addition, this study also suggests that the operational criteria proposed by the RSWG might define a subgroup of patients with milder core symptoms since their first episode of illness, and, hence, they could have a higher probability to achieve long-term remission. Therefore, the RSWG remission criteria (Andreasen et al., 2005) could tell more about severity of the core symptoms at first episode, than about remission itself. This conclusion is particularly true for negative symptoms, since the more severe is the social isolation at the first episode, the less probable the achievement of long-term remission.

The present study has the following limitations. First, the symptoms severity was evaluated at onset and after a mean period of 16 years of illness. However we can exclude that deep fluctuation of symptom severity may occur during the course of illness, since symptoms severity was clinically monitored nearly at monthly visits. Nonetheless, since schizophrenia is not a stable disorder and remission, as well as recovery, is for many patients a time limited occurrence, we cannot exclude that possible relapses may occur. Second, even though the treatment was clinically monitored at each visit and strategies to prevent non-adherence were planned during the follow-up period, we cannot rule out that some patients were non-adherent to medications between the monthly visits.

In conclusion, according to the data of the present study, longterm symptomatic remission, even though wished for all schizophrenic patients, would seem a viable therapeutic outcome 
particularly in patients with less severe core symptoms, particularly negative symptoms, at their first episode.

\section{References}

Addington, J., Addington, D., 2008. Symptom remission in first episode patients. Schizophrenia Research 106, 281-285.

Allen, D.N., Franton, L.V., Strauss, G.P., van Kammen, D.P., 2005. Differential patterns of premorbid academic and social deterioration in patients with schizophrenia. Schizophrenia Research 75, 389-397.

Amaddeo, F., Barbui, C., Tansella, M., 2012. State of psychiatry in Italy 35 years after psychiatric reform. A critical appraisal of national and local data. International Review of Psychiatry 24, 314-320.

Andreasen, N.C., Carpenter, W.T., Kane, J.M., Lasser, R.A., Marder, S.R., Weinberger, D.R., 2005. Remission in schizophrenia: proposed criteria and rationale for consensus. The American Journal of Psychiatry 162, 441-449.

Ayesa-Arriola, R., Rodríguez-Sánchez, J.M., Pérez-Iglesias, R., González-Blanch, C., PardoGarcía, G. Tabares-Seisdedos, R., Vàzquez-Barquero, J.L., Crespo-Facorro, B., 2013. The relevance of cognitive, clinical and premorbid variables in predicting functional outcome for individuals with first-episode psychosis: a 3 year longitudinal study. Psychiatry Research 209, 302-308.

Bleuler, E., 1911. Dementia praecox oder Gruppe der Schizophrenien. Deutcke, Leipzig.

Buoli, M., Caldiroli, A., Panza, G., Altamura, A.C., 2012. Prominent clinical dimension, duration of illness and treatment response in schizophrenia: a naturalistic study. Psychiatry Investigation 9, 354-360.

Chang, W.C., Tang, J.Y., Hui, C.L., Wong, G.H., Chan, S.K., Lee, E.H., Chen, E.Y., 2013. The relationship of early premorbid adjustment with negative symptoms and cognitive functions in first-episode schizophrenia: a prospective three-year follow-up study. Psychiatry Research 209, 353-360.

Crow, T.J., 1985. The two-syndrome concept: origins and current status. Schizophrenia Bulletin 11, 471-486.

Karow, A., Moritz, S., Lambert, M., Schöttle, D., Naber, D., 2012. Remitted but still impaired? Symptomatic versus functional remission in patients with schizophrenia. European Psychiatry 27, 401-405.

Kirkpatrick, B., Buchanan, R.W., Ross, D.E., Carpenter, W.T., 2001. A separate disease within the syndrome of schizophrenia. Archives of General Psychiatry 58, 165-171.

Kraepelin, E., 1899. Ein Lehrbuch für Studierende und Äertze (Auflage Psychiatrie). Johann Ambrosins Barth, Leipzig, Austria p. 6.
Kurihara, T., Kato, M., Reverger, R., Tirta, I.G., 2011. Remission in schizophrenia: a community-based 6-year follow-up study in Bali. Psychiatry and Clinical Neuroscience 65, 476-482.

Lambert, M., Schimmelmann, B.G., Naber, D., Schacht, A., Karow, A., Wagner, T., Czekalla, J., 2006. Prediction of remission as a combination of symptomatic and functional remission and adequate subjective well-being in 2960 patients with schizophrenia. Journal of Clinical Psychiatry 67, 1690-1697.

Lambert, M., De Marinis, T., Pfeil, J., Naber, D., Schreiner, A., 2010. Establishing remission and good clinical functioning in schizophrenia: predictors of best outcome with long-term risperidone long-acting injectable treatment. European Psychiatry 25, 220-229.

Lang, F.U., Kösters, M., Lang, S., Becker, T., Jäger, M., 2013. Psychopathological longterm outcome of schizophrenia-a review. Acta Psychiatrica Scandinavica 127, $173-182$.

Lasser, R.A., Nasrallah, H., Helldin, L., Peuskens, J., Kane, J., Docherthy, J., Tronco, A.T. 2007. Remission in schizophrenia: applying recent consensus criteria to refine the concept. Schizophrenia Research 96, 223-231.

Marchesi, C., Affaticati, A., Monici, A., De Panfilis, C., Ossola, P., Tonna, M., 2014 Predictors of symptomatic remission in patients with first-episode schizophrenia: a 16years follow-up study. Comprehensive Psychiatry 55, 778-784.

McGlashan, T.H., 2008. Premorbid adjustment, onset types, and prognostic scaling: still informative? Schizophrenia Bulletin 34, 801-805.

Novick, D., Haro, J.M., Suarez, D., Lambert, M., Naber, D., 2007. Symptomatic remission in previously untreated patients with schizophrenia: 2-year results from the SOHO study. Psychopharmacology 191, 1015-1022.

Rossi, A., Bagalà, A., Del Curatolo, V., Scapati, F., Bernareggi, M.M., Giustra, M.G. 2009. Remission in schizophrenia: one-year Italian prospective study of risperidone long-acting injectable (RLAI) in patients with schizophrenia or schizoaffective disorder. Human Psychopharmacology 24, 574-583.

San, L., Ciudad, A., Alvarez, E., Bobes, J., Gilaberte, I., 2007. Symptomatic remission and social/vocational functioning in outpatients with schizophrenia: prevalence and associations in a cross-sectional study. European Psychiatry 22 490-498.

Strous, R.D., Alvir, J.M.J., Robinson, D., Gal, G., Sheitman, B., Chakos, M., Lieberman, J.A., 2004. Premorbid functioning in schizophrenia: relation to baseline symptoms, treatment response, and medication side effects. Schizophrenia Bullettin 30, 265-278.

Ventura, J., Hellemann, G.S., Thames, A.D., Joellner, V., Nuechterlein, K.H., 2009 Symptoms as mediators of the relationship between neurocognition and functional outcome in schizophrenia. A meta-analysis. Schizophrenia Research 113, 189-199.

Wunderink, L., Sytema, S., Nienhuis, F.J., Wiersma, D., 2009. Clinical recovery in first-episode psychosis. Schizophrenia Bulletin 35, 362-369. 